\title{
THE SUBDIRECT DECOMPOSITION THEOREM FOR CLASSES OF STRUCTURES CLOSED UNDER DIRECT LIMITS
}

\author{
XAVIER CAICEDO \\ (Received 16 March 1979; revised 24 January 1980) \\ Communicated by D. E. Taylor
}

\begin{abstract}
By a theorem of G. Birkhoff, every algebra in an equationally defined class of algebras $K$ is a subdirect product of subdirectly irreducible algebras of $K$. In this paper we show that this result is true for any class of structures. not necessarily algebraic, closed under isomorphisms and direct limits. Quasivarieties in the sense of Makev are examples of such classes of structures. This includes Birkhoffs result as a particular case.
\end{abstract}

1980. Mathematics subject classification (Amer. Math. Soc.) : primary 08 A 05; secondary 08 C 15,03 C 52.

\section{Introduction}

A well-known theorem of Birkhoff (1944) states that if $K$ is an equationally defined class of algebras (a variety) then every algebra in $K$ is a subdirect product of subdirectly irreducible algebras of $K$. From this result many representation theorems follow, including Stone's representation theorem for Boolean Algebras and representation theorems for Abelian Groups, see Pierce (1968). This theorem is also important in Algebraic Logic since completeness of a logic with respect to a given semantics follows sometimes from a representation theorem as in Halmos (1962) and Rasiowa (1974).

We are interested in knowing if the subdirect decomposition theorem is true for quasivarieties in the sense of Malcev (1971). It is shown in this paper that the answer is positive, not only for quasivarieties but for any class of structures closed under isomorphisms and direct limits. In particular, Birkhoff's theorem may be shown without using the notion of congruence relation. 
In Sections 1 and 3, we recall the basic notions in the framework of an arbitrary class of structures. In the second section we characterize the subdirectly irreducible structures. In Section 4 we show the existence of enough of such structures for classes closed under direct limits. The main theorem appears in Section 5.

\section{Subdirect products}

We assume that all structures mentioned in this work are of a fixed similarity type $\tau$. The equality symbol together with the relation symbols of $\tau$ are called the basic predicates. If $\mathfrak{H}$ is a structure then $|\mathfrak{H}|$ denotes its universe and $\|\mathfrak{H}\|$ denotes its cardinal. If $P$ and $f$ are, respectively, a basic predicate and a function symbol of $\tau$. then $P^{\mathscr{\mu l}}$ and $f^{\mathscr{\mu}}$ are their interpretations in $\mathscr{H}$. The interpretation of the equality symbol is always equality. We recall some basic definitions. Let $\mathfrak{U}$ and $\mathfrak{B}$ be structures, $\varphi:|\mathfrak{U}| \rightarrow|\mathfrak{B}|$ a function.

(1) $\varphi$ is an homomorphism, denoted $\varphi: \mathscr{U} \rightarrow \mathfrak{B}$, if (i) for all $n$-adic basic predicate $P$. $\left(a_{1}, \ldots, a_{n}\right) \in P^{* 1}$ implies $\left(\varphi\left(a_{1}\right), . ., \varphi\left(a_{n}\right)\right) \in P^{\mathfrak{B}}$ and (ii) for all $n$-adic function symbol $f$.

$$
\varphi\left(f^{\prime \prime \prime}\left(a_{1}, \ldots, a_{n}\right)\right)=f^{\mathfrak{B}}\left(\varphi\left(a_{1}\right), \ldots, \varphi\left(a_{n}\right)\right) .
$$

(2) $\varphi$ is a substructure monomorphism if it is an homomorphism with condition (ii) in (1) strengthenéd to : (ii*) for all $a_{1}, \ldots, a_{n}$ in $|\mathfrak{U}|$ and all $n$-adic basic predicate $P$, $\left(a_{1}, \ldots, a_{n}\right) \in P^{* 1}$ if and only if $\left(\varphi\left(a_{1}\right), \ldots, \varphi\left(a_{n}\right)\right) \in P^{p R}$.

A substructure monomorphism must be a one to one homomorphism. However, the converse implication is not valid. $\mathscr{U}$ is a substructure of $\mathfrak{B}$ if $|\mathfrak{U}| \subseteq|\mathfrak{B}|$ and the inclusion function is a substructure monomorphism.

(3) $\varphi$ is an isomorphism if it is an onto substructure monomorphism. As is well known, $\varphi$ is an isomorphism if and only if there is an homomorphism $\psi: \mathfrak{B} \rightarrow \mathfrak{U}$ such that $\varphi \psi=1_{\mathfrak{y}}$ and $\psi \varphi=1_{\mathfrak{B}}$, the identity homomorphisms of $\mathscr{U}$ and $\mathfrak{B}$, respectively. Note that $\varphi: \mathfrak{U} \rightarrow \mathfrak{B}$ is a substructure monomorphism if and only if there is a substructure $\mathfrak{B}^{\prime}$ of $\mathfrak{B}$ such that $\varphi:|\mathfrak{H}| \rightarrow\left|\mathfrak{B}^{\prime}\right|$ is an isomorphism from $\mathscr{U}$ to $\mathfrak{B}^{\prime}$.

(4) Let $\left\{\boldsymbol{B}_{i} \mid i \in I\right\}$ be a family of structures. Let $B^{*}$ be the usual cartesian product of the family of sets $\left\{\left|\mathfrak{B}_{i}\right|: i \in I\right\}$ and let $\pi_{j}: B^{*} \rightarrow\left|\mathfrak{B}_{j}\right|$ be the $j$-projection. The (cartesian) product of the family of structures is the structure $\Pi \mathfrak{B}_{i}=\left\langle B^{*}, \ldots, P^{*}, \ldots, f^{*}\right\rangle$ where :

(i) $\left(a_{1}, \ldots, a_{n}\right) \in P^{*}$ if and only if $\forall i \in I,\left(\pi_{i}\left(a_{1}\right), \ldots, \pi_{i}\left(a_{n}\right)\right) \in P^{*_{4}}$,

(ii) $\forall i \in I, f^{*}\left(a_{1}, \ldots, a_{n}\right)(i)=f^{\mathcal{B}}\left(a_{1}, \ldots, a_{n}\right)$.

The projections become onto homomorphisms $\pi_{j}: \Pi \mathfrak{B}_{i} \rightarrow \mathfrak{B}_{j}$ provided $\Pi \mathfrak{B}_{i}$ is not empty. Moreover, the product has the following universal property. If $\left\{f_{i}: \mathfrak{U} \rightarrow \mathfrak{B}_{i} \mid i \in \mathbf{I}\right\}$ is a family of homomorphisms, there exists a unique homomorphism $g: \mathfrak{U} \rightarrow \Pi \mathfrak{B}_{i}$ such that $f_{i}=\pi_{i} g$ for all $i \in I$. Uniqueness follows because the 
last condition forces $g(a)(i)=f_{i}(a)$. It is routine to check that this definition gives an homomorphism. The product is characterized up to isomorphism by the above universal property. In other words, if the family $\left\{p_{i}: \mathfrak{B} \rightarrow \mathfrak{B}_{i} \mid i \in I\right\}$ is such that for every family $\left\{f_{i}: \mathfrak{U} \rightarrow \mathfrak{B}_{i} \mid i \in I\right\}$ there is a unique $g: \mathfrak{U} \rightarrow \mathfrak{B}$ with $f_{i}=p_{i} g$ for all $i \in I$, then there is an isomorphism $h: \mathfrak{B} \rightarrow \Pi^{\mathfrak{B}_{i}}$ such that for all $i \in I, p_{i}=\pi_{i} h$.

Now we introduce the notion of subdirect product, generalizing the definition usually given for algebras, as in Grätzer (1968) or Pierce (1968).

Definition 1. Let $\left\{\mathfrak{B}_{i} \mid i \in I\right\}$ be a family of structures. A subdirect product of the family is a substructure monomorphism $\varphi: \mathfrak{U} \rightarrow \Gamma^{\mathfrak{B}_{i}}$ such that $\pi_{i} \varphi$ is onto for every $i \in I$. It is called also a subdirect decomposition of $\mathfrak{U}$.

For example, if $\mathfrak{B}_{i}=\mathscr{U}$ for all $i \in I$, then the diagonal substructure $\Delta: \mathfrak{U} \rightarrow \prod \mathfrak{B}_{i}$, where $\Delta(a)(i)=a$ for all $i$ is a subdirect product. In general, one may obtain trivial subdirect decompositions of $\mathfrak{U}$ in the following way. Let $\left\{f_{i}: \mathfrak{U} \rightarrow \mathfrak{B}_{i} \mid i \in I\right\}$ be a family of onto homomorphisms that contains at least one isomorphism $f_{r}$. Let $\varphi$ be the map induced by the $f_{i}$ 's into $\prod \mathfrak{B}_{i}$. Then $\varphi$ is a substructure monomorphism because $\left(\varphi\left(a_{1}\right), \ldots, \varphi\left(a_{n}\right)\right) \in P^{*}$ implies

$$
\left(f_{r}\left(a_{1}\right), \ldots, f_{r}\left(a_{n}\right)\right)=\left(\pi_{r} \varphi\left(a_{1}\right), \ldots, \pi_{r} \varphi\left(a_{n}\right)\right) \in P^{\mathfrak{B} r}
$$

and so $\left(a_{1}, \ldots, a_{n}\right) \in P^{* \prime}$, since $f_{r}$ is an isomorphism. Moreover, $\pi_{i} \varphi=f_{i}$ is onto for each $i \in I$; hence $\varphi$ is a subdirect product of $\left\{\mathfrak{B}_{i} \mid i \in I\right\}$. The first example above is of this kind. A structure is subdirectly irreducible if all its subdirect decompositions are trivial in this sense. Relativizing this notion to a class $K$ of structures closed under isomorphisms, we have :

Definition 2. $\mathscr{U}$ is subdirectly irreducible (s.i.) in $K$, if for every subdirect decomposition $\varphi: \mathfrak{U} \rightarrow \prod^{\mathfrak{B}_{i}}$ with all $\mathfrak{B}_{i}$ 's in $K$, there is $j \in I$ such that $\pi_{j} \varphi$ is an isomorphism.

Birkhoff has shown that if $K$ is an equationally defined class of algebras, then the s.i. algebras of $K$ are those for which the intersection of their nontrivial congruence relations is nontrivial. Moreover, for any algebra $\mathscr{U}$ of $K$ there is a subdirect product $\varphi: \mathfrak{U I} \rightarrow \Pi^{\mathfrak{B}_{i}}$ where the $\mathfrak{B}_{i}$ 's are all s.i. algebras of $K$. For example, if $K$ is the class of Boolean Algebras the only s.i. algebra of $K$ is the two element boolean algebra $\mathbf{2}$, and each boolean algebra is a subalgebra of a cartesian product of copies of 2 (Stone's theorem). If $K$ is the class of Abelian Groups the s.i. algebras are the $\mathbf{Z}_{p^{n}}$ and $\mathbf{Z}_{p^{\prime}}$ for $p$ prime (Pierce (1968), pp. 52-56). In the following section we give a characterization of s.i. structures in an arbitrary class $K$. Later, we shall generalize the subdirect decomposition theorem. 


\section{Characterization of subdirectly irreducible structures}

In the rest of this work, we assume that $K$ is a class of structures closed under isomorphisms.

LEMMA 1. $\mathfrak{U}$ is subdirectly irreducible in $K$ if and only if there exists an n-adic basic predicate $P$ and an n-tuple $\left(a_{1}, \ldots, a_{n}\right) \in|\mathfrak{U}|^{n}$ such that $\left(a_{1}, \ldots, a_{n}\right) \notin P^{2}$, and for every onto homomorphism $f: \mathfrak{U} \rightarrow \mathfrak{B}$ with $\mathfrak{B}$ in $K$, which is not an isomorphism, $\left(f\left(a_{1}\right), \ldots, f\left(a_{n}\right)\right) \in P^{b}$.

Proof. Let $P$ and $\left(a_{1}, \ldots, a_{n}\right)$ have the property indicated in the statement of the lemma. Let $\varphi: \mathscr{H} \rightarrow \Pi \mathfrak{B}_{i}$ be a subdirect product. Since $\left(a_{1}, \ldots, a_{n}\right) \notin P^{* 1}$ and $\varphi$ is a substructure monomorphism then $\left(\varphi\left(a_{1}\right), \ldots, \varphi\left(a_{n}\right)\right) \notin P^{*}$. Hence,

$$
\left(\pi_{i} \varphi\left(a_{i}\right), \ldots, \pi_{i} \varphi\left(a_{n}\right)\right) \notin P^{* \psi_{i}}
$$

for some $i \in I$. By hypothesis and the fact that $\pi_{i} \varphi$ is onto, we conclude that $\pi_{i} \varphi$ is an isomorphism; this shows that $\varphi$ is subdirectly irreducible.

Conversely, suppose $\mathfrak{U}$ is subdirectly irreducible. Let $C=\{(f, \mathfrak{B}) \mid f: \mathfrak{U} \rightarrow \mathfrak{B}$ is an onto homomorphism but not an isomorphism and $\mathfrak{B} \in K\}$. It is a proper class. Define $(f, \mathfrak{B}) \sim\left(f^{\prime}, \mathfrak{B}^{\prime}\right)$ if and only if there is an isomorphism $g: \mathfrak{B} \rightarrow \mathfrak{B}^{\prime}$ such that $f^{\prime}=g f$. There is only a set of nonisomorphic structures of cardinality $\leqslant\|\mathfrak{U}\|$ and only a set of homomorphisms from $\mathscr{U}$ to $\mathfrak{B}$ for each possible $\mathfrak{B}$. Thus $\sim$ is an equivalence relation in $C$ which has a set of representatives, since if $f: \mathfrak{U} \rightarrow \mathfrak{B}$ is onto, then $\|\mathfrak{U}\| \leqslant\|\mathfrak{B}\|$.

Let $F=\left\{\left(f_{i}, \mathfrak{B}_{i}\right) \mid i \in I\right\}$ be a family of representatives. Take the product $\Pi \mathfrak{B}_{i}$ and the homomorphism $g: \mathfrak{U} \rightarrow \prod \mathfrak{B}_{i}$ induced by the $f_{i}$ 's. Since $\mathfrak{U}$ is subdirectly irreducible and each $\pi_{i} g=f_{i}$ is not an isomorphism, $g$ is not a subdirect product. But each $\pi_{i} g$ is onto, so $g$ cannot be a substructure monomorphism. This means that there exist a predicate $P$ (may be equality) and an $n$-tuple $\left(a_{1}, \ldots, a_{n}\right)$ such that $\left(g\left(a_{1}\right), \ldots, g\left(a_{n}\right)\right) \in P^{*}$, but $\left(a_{1}, \ldots, a_{n}\right) \notin P^{\mathscr{l}}$. Now, let $f: \mathfrak{U} \rightarrow \mathfrak{B}$ be any onto homomorphism, $\mathfrak{B} \in K$, that is not an isomorphism. Then $(f, \mathfrak{B})$ has a representative in $F$ and so $f=h f_{i}$ for some isomorphism $h$ and $i \in I$. Therefore, $f=h \pi_{i} g$. Since $\left(g\left(a_{1}\right), \ldots, g\left(a_{n}\right)\right) \in P^{*}$ and $h \pi_{i}$ is homomorphism it follows that

$$
\left(f\left(a_{1}\right), \ldots, f\left(a_{n}\right)\right)=\left(h \pi_{i} g\left(a_{1}\right), \ldots, h \pi_{i} g\left(a_{n}\right)\right) \in P^{\mathfrak{B}} .
$$

We have shown that $\left(a_{1}, \ldots, a_{n}\right) \notin P^{\Downarrow}$, but for any homomorphism $f: \mathscr{U} \rightarrow \mathfrak{B}(\mathfrak{B} \in K)$ which is not isomorphism, $\left(f\left(a_{1}\right), \ldots, f\left(a_{n}\right)\right) \in P^{\mathfrak{B}}$.

COROLlary (Birkhoff). An algebra of an equationally defined class $K$ is s.i. in $K$ if and only if the intersection of all its non-trivial congruence relations is non tritial (distinct from the diagonal). 
PROOF. The only basic predicate of $\mathscr{U}$ is ' $=$ ' so by Lemma 1 there are elements $a, b$ in $\mathscr{U}$ such that $a \neq b$ and for any onto homomorphism $f: \mathfrak{U} \rightarrow \mathfrak{B}$ which is not an isomorphism, $f(a)=f(b)$ or $(a, b) \in \operatorname{Congr}(f)$. By the correspondence between congruence relations and onto homomorphisms $(a, b)$ belongs to all nontrivial congruence relations of $\mathfrak{U}$.

\section{Direct limits}

A partially ordered set $\langle\Sigma . \leqslant\rangle$ is directed fif for all $i, j$ in $\Sigma$ there is $k$ in $\Sigma$ such that $i \leqslant k$ and $j \leqslant k$. A directed diagram of structures over $\langle\Sigma, \leqslant\rangle$ is a family of structures $\left\{\mathfrak{B}_{i} \mid i \in \Sigma\right\}$ and a family of homomorphisms $\left\{f_{i j}: \mathfrak{B}_{i} \rightarrow \mathfrak{B}_{j} \mid i \leqslant j\right\}$ such that $f_{i i}=1_{\mathfrak{B}_{i}}$ and for all $i \leqslant j \leqslant k, f_{i k}=f_{j k} f_{i j}$.

DefinImon 3. Let $D=\left\langle\left\{\mathfrak{B}_{i} \mid i \in \Sigma\right\},\left\{f_{i j} \mid i \leqslant j\right\}\right\rangle$ be a directed diagram. The direct limit of $D$ is the structure $\lim _{\rightarrow} D=\langle\underset{B}{\rightarrow}, \ldots, \underline{P}, \ldots, f, \ldots$.$\rangle where$

(i) $\stackrel{B}{\rightarrow}=\amalg\left|\mathfrak{B}_{i}\right| / \sim$, the disjoint union of the $\left|\overrightarrow{B_{i}}\right|$ 's divided by the equivalence relation :

$$
a \sim a^{\prime} \quad \text { if and only if } \exists k \geqslant i, j \text { such that } f_{i k}(a)=f_{j k}\left(a^{\prime}\right) .
$$

(ii) If $\bar{a}$ denotes the equivalence class of $a$ in $\amalg\left|\mathfrak{B}_{i}\right|$, then for all $\bar{a}_{1}, \ldots, \bar{a}_{n}$ in $B$ with $a_{r}$ in $\left|\mathfrak{B}_{i r}\right|$ we have :

(a) $\left(a_{1}, \ldots, a_{n}\right) \in P$ if and only if $\exists k \geqslant i_{1}, \ldots, i_{n}$ such that

$$
\left(f_{i, k}\left(a_{1}\right), \ldots, f_{i n k}\left(a_{n}\right)\right) \in P^{\mathfrak{B}_{k}} \text {. }
$$

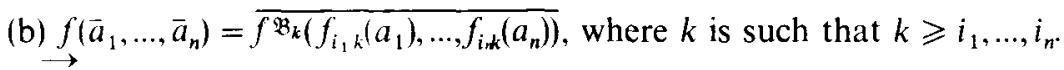

DEFINITION 4. For each $i \in \Sigma$ the canonical homomorphism $g_{i}: \mathfrak{B}_{i} \rightarrow \lim _{\rightarrow} D$ is defined by $g_{i}(a)=\bar{a}$.

It is a tedious exercise to show that $\sim$ is in fact an equivalence relation, $P$ and $f$ are well defined, and the $g_{i}$ 's are homomorphisms. See Grätzer (1968), p. 128, for the case of algebras. The following universal property characterizes the family $\left\{g_{i}: \mathfrak{B}_{i} \rightarrow \lim D\right\}$ up to isomorphism :

For all $i, j$ in $\Sigma$ with $i \leqslant j, g_{j} f_{i j}=g_{i}$, and given any family of homomorphisms $\left\{h_{i}: \mathfrak{B}_{i} \rightarrow \mathfrak{C} \mid i \in \Sigma\right\}$ such that $h_{j} f_{i j}=h_{i}$ for all $i \leqslant j$, there exists a unique homomorphism $\delta: \lim D \rightarrow \mathfrak{C}$ such that $h_{i}=\delta g_{i}$ for all $i \in \Sigma$. (Let $a \in\left|\mathfrak{B}_{i}\right|$, then define $\delta(a)=h_{i}(a)$. It is routine to check that $\delta$ is well defined and has the desired properties.) 
The important property of directed limits that we shall use is the following :

LEMMA 2. Let $D=\left\langle\left\{\mathfrak{B}_{i} \mid i \in \Sigma\right\},\left\{f_{i j} \mid i \leqslant j\right\}\right\rangle$ be a directed diagram, and fix $i \in \Sigma$. Iff is onto for all $k \geqslant i$ then the canonical homomorphism $y_{i}$ is onto.

PROOF. If $x \in\left|\lim _{\rightarrow} D\right|$, then $x=\bar{a}$ with $a \in\left|\mathfrak{B}_{j}\right|$ for some $j \in \Sigma$. Choose $k \geqslant i, j$ then $x=\bar{a}=g_{j}(a)=\vec{g}_{k} f_{j k}(a)$. But $f_{i k}$ is onto and $f_{j k}(a)=f_{i k}\left(a^{\prime}\right)$ for some $a^{\prime} \in\left|\mathfrak{B}_{i}\right|$. Hence, $x=g_{k} f_{j k}(a)=g_{k} f_{i k}\left(a^{\prime}\right)=g_{i}\left(a^{\prime}\right)$.

Another important observation is the following. If $\left(a_{i}, \ldots, a_{n}\right) \in\left|\mathfrak{B}_{i}\right|^{n}$ and $P$ is a basic $n$-adic predicate, then $\left(g_{i}\left(a_{1}\right), \ldots, g_{i}\left(a_{n}\right)\right) \in P$ if and only if $\left(f_{i k}\left(a_{1}\right), \ldots, f_{i k}\left(a_{n}\right)\right) \in P^{\mathfrak{B}_{k}}$ for some $k \geqslant i$. This follows from Definition 3 (ii) (a), and Definition 4.

\section{Existence of subdirectly irreducible structures}

If $K$ is closed under limits it has a good supply of subdirectly irreducible structures. Actually, closure under direct limits of families of onto homomorphisms suffices.

LEMMA 3. Let $K$ be closed under direct limits of families of onto homomorphisms. Let $\mathfrak{U} \in K,\left(a_{1}, \ldots, a_{n}\right) \in|\mathfrak{U}|^{n}$, and $P$ be a basic $n$-adic predicate such that $\left(a_{1}, \ldots, a_{n}\right) \notin P^{* !}$. Then there exists $\mathfrak{B} \in K$, subdirectly irreducible in $K$, and an onto homomorphism $f: \mathfrak{U} \rightarrow \mathfrak{B}$ such that $\left(f\left(a_{1}\right), \ldots, f\left(a_{n}\right)\right) \notin P^{\mathfrak{B}}$.

ProOF. Given onto homomorphisms $f: \mathscr{U} \rightarrow \mathfrak{B}$ and $f^{\prime}: \mathfrak{U} \rightarrow \mathfrak{B}^{\prime}$ define $(f, \mathfrak{B}) \sim\left(f^{\prime}, \mathfrak{B}^{\prime}\right)$ if and only if there exists an isomorphism $g: \mathfrak{B} \rightarrow \mathfrak{B}$ such that $f^{\prime}=g f$. This is obviously an equivalence relation in the class of all pairs $(f, \mathfrak{B})$ with $f: \mathfrak{U} \rightarrow \mathfrak{B}$ an onto homomorphism. As shown in the proof of Lemma 1 , this equivalence relation has a set of representatives for its equivalence classes. Let $R$ be a set of representatives and let

$$
F=\left\{(f, \mathfrak{B}) \in R \mid \mathfrak{B} \in K,\left(f\left(a_{1}\right), \ldots, f\left(a_{n}\right)\right) \notin P^{\mathfrak{B}}\right\} .
$$

We define a partial order in $F$ by

$$
(f, \mathfrak{B})<\left(f^{\prime}, \mathfrak{B}^{\prime}\right) \text { if and only if there is } g \text { such that } f^{\prime}=g f .
$$

Reflexivity and transitivity are obvious. Now, if $(f, \mathfrak{B})<\left(f^{\prime}, \mathfrak{B}^{\prime}\right)$ and $\left(f^{\prime}, \mathfrak{B}^{\prime}\right) \prec(f, \mathfrak{B})$ then $f^{\prime}=g f$ and $f=h f^{\prime}$ for some homomorphism $g$ and $h$. Therefore, $f^{\prime}=g h f^{\prime}$ and $f=h g f$. Since $f$ and $f^{\prime}$ are onto, $g h=1_{\mathfrak{B}}$ and $h g=1_{\mathfrak{B}}$ and this shows that $g$ is an isomorphism. Hence, $(f, \mathfrak{B}) \sim\left(f^{\prime}, \mathfrak{B}^{\prime}\right)$ and $(f, \mathfrak{B})=\left(f^{\prime}, \mathfrak{B}^{\prime}\right)$. This shows antisymmetry. 
Now, we will apply Zorn's lemma to show that $F$ has maximal elements. Given a chain in $F,\left\{\left(f_{i}, \mathfrak{B}_{i}\right) \mid i \in I\right\}$ with $I$ totally ordered, there is for every $i, j \in I$ with $i \leqslant j$ an homomorphism $f_{i j}: \mathfrak{B}_{i} \rightarrow \mathfrak{B}_{j}$ such that $f_{j}=f_{i j} f_{i}$. Actually $f_{i j}$ is unique because $f_{i}$ is onto

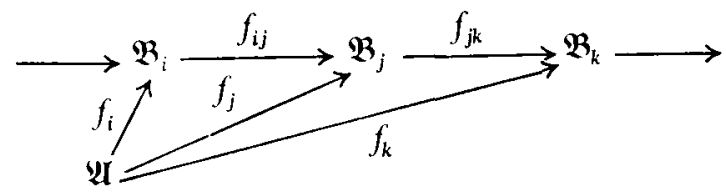

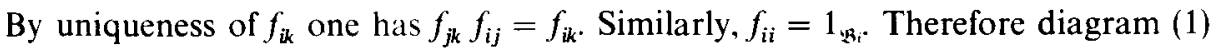
above is directed over the directed set $\{0\} \cup I$ (make $0<i, \mathfrak{B}_{0}=\mathscr{U}$, and $f_{0 i}=f_{i}$ for $i \in I$ ). Moreover, every morphism in the diagram is onto $\left(f_{i j}\right.$ is onto because $f_{j}$ is). Let $\mathfrak{D}$ be a direct limit of the diagram, $\mathcal{D} \in K$, and let $g_{i}: \mathfrak{B}_{i} \rightarrow \mathfrak{D}, f: \mathfrak{U} \rightarrow \mathfrak{D}$ be the canonical homomorphisms. By Lemma $2, f$ is onto. Therefore, we may assume that $(f, \mathfrak{D}) \in R$. To conclude that $(f, \mathfrak{D}) \in F$ we must show that $\left(f\left(a_{1}\right), \ldots, a\left(a_{n}\right)\right) \notin P^{\mathfrak{D}}$. Suppose $\left(f\left(a_{1}\right), \ldots, f\left(a_{n}\right)\right) \in P^{\mp}$, then for some morphism $f_{k}$ in the diagram

$$
\left(f_{k}\left(a_{1}\right), \ldots, f_{k}\left(a_{n}\right)\right) \in P^{\mathfrak{R}_{k}}
$$

which contradicts the way the $\mathfrak{B}_{i}$ 's were chosen. Finally, $\left(f_{i}, \mathfrak{B}_{i}\right) \prec(f, \mathfrak{B})$ because $g_{i} f_{i}=g_{i} f_{0 i}=g_{0}=f$.

We have shown that the chain has an upper bound. Let $(f, \mathfrak{B})$ be a maximal element of $F$, then $\left(f\left(a_{1}\right), \ldots, f\left(a_{n}\right)\right) \notin P^{\mathfrak{B}}$. Now let $g: \mathfrak{B} \rightarrow \mathfrak{B}^{\prime}$ be any onto homomorphism with $\mathfrak{B}^{\prime} \in K$ and $\left(g\left(f\left(a_{1}\right)\right), \ldots, g\left(f\left(a_{n}\right)\right)\right) \notin P^{\mathfrak{B}^{\prime}}$. Then $\left(g f, \mathfrak{B}^{\prime}\right)$ must have a representative $\left(f^{\prime \prime}, \mathfrak{B}^{\prime \prime}\right)$ in $F$ and the following diagram, where $h$ is an isomorphism, commutes :

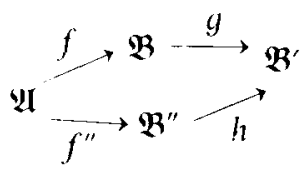

Since $f^{\prime \prime}=h^{-1} g f$ it follows that $(f, \mathfrak{B}) \prec\left(f^{\prime \prime}, \mathfrak{B}^{\prime \prime}\right)$ and by maximality $(f, \mathfrak{B})=\left(f^{\prime \prime}, \mathfrak{B} \prime\right)$. Therefore. $h f=h f^{\prime \prime}=g f$. But $f$ is onto so $g=h$ is an isomorphism. This shows that for any onto homomorphism $g: \mathfrak{B} \rightarrow \mathfrak{B}^{\prime}$ which is not an isomorphism we must have $\left(g\left(f\left(a_{1}\right), \ldots, g\left(f\left(a_{n}\right)\right) \in P^{\mathfrak{B}^{\prime}}\right.\right.$. Hence, $\mathfrak{B}$ is subdirectly irreducible by Lemma 1.

\section{Subdirect decomposition theorem}

Let $\|\tau\|$ be the number of basic predicates in $\tau$.

THEOREM 4. Let $K$ be closed under direct limits (of onto homomorphisms), then every structure $\mathscr{U} \in K$ is a subdirect product of at most $\aleph_{0}+\|\mathfrak{U}\|+\|\tau\|$ subdirectly irreducible structures in $K$. 
Proof. Let $\mathscr{U} \in K$. Let $F=\left\{\left(f_{i}, \mathfrak{B}_{i}\right) \mid i \in I\right\}$ be a family such that (i) $f_{i}: \mathscr{U} \rightarrow \mathfrak{B}_{i}$ is an onto homomorphism, (ii) $\mathfrak{B}_{i} \in K$, (iii) $\mathfrak{B}_{i}$ is subdirectly irreducible, and (iv) for each $n$ adic basic predicate $P$ (including equality) and $n$-tuple $\left(a_{1}, \ldots, a_{n}\right) \in|\mathscr{U}|^{n}$ such that $\left(a_{1}, \ldots, a_{n}\right) \notin P^{\Downarrow \prime \prime}$, there exists $i \in I$ for which $\left(f_{i}\left(a_{1}\right), \ldots, f_{i}\left(a_{n}\right)\right) \notin P^{\mathfrak{B}_{i}}$. A family like this exists by Lemma 3 , and it is clear that it may be chosen of cardinality at most

$$
\sum_{n}\|\mathfrak{U}\|^{n}\|\tau\| \leqslant \boldsymbol{N}_{0}+\|\mathfrak{U}\|+\|\tau\| \text {. }
$$

The family of homomorphisms $\left\{f_{i} \mid i \in I\right\}$ induces an homomorphism $g$ from $\mathcal{U}$ into the cartesian product $\Pi \mathfrak{B}_{i}$ such that $\pi_{j} g=f_{j}$ for each $i \in I$. This homomorphism is actually an isomorphism into a substructure of $\Gamma \mathfrak{B}_{i}$ because for every basic predicate $P$ and $n$-tupule $\left(a_{1}, \ldots, a_{n}\right)$ we have:

$$
\begin{aligned}
\left(g\left(a_{1}\right), \ldots, g\left(a_{n}\right)\right) \in P^{*} & \Leftrightarrow\left(\pi_{i} g\left(a_{1}\right), \ldots, \pi_{i} g\left(a_{n}\right)\right) \in P^{\mathfrak{B}_{i}} \text { for all } i \in I \\
& \Leftrightarrow\left(f_{i}\left(a_{1}\right), \ldots, f_{i}\left(a_{n}\right)\right) \in P^{* 3_{i}} \text { for all } i \in I \\
& \Rightarrow\left(a_{1}, \ldots, a_{n}\right) \in P^{* !}
\end{aligned}
$$

The last implication being true because of condition (iv) in the definition of $F$. Since each $\pi_{i} g=f_{i}$ is onto by construction, this finishes the proof.

COROllary 5. Let $K$ be a class of structures axiomatized by sentences of the form:

$$
\forall x_{1} \ldots \forall x_{n}\left(\Phi \rightarrow \exists y_{1} \ldots \exists y_{t} \Theta\right),
$$

where $\Phi$ and $\Theta$ are quantifier-free positive formulae, then every structure in $K$ is a subdirect product of subdirectly irreducible structures of $K$.

Proof. It is enough to show that sentences of the above form are preserved by direct limits. Let $\left\langle\left\{\mathfrak{B}_{i} \mid i \in \sum\right\},\left\{f_{i j}: \mathfrak{B}_{i} \rightarrow \mathfrak{B}_{j}\right\}\right\rangle$ be a directed diagram over $\langle\Sigma, \leqslant\rangle$. with direct limit $\left\{g_{i}: \mathfrak{B}_{i} \rightarrow \mathfrak{D} \mid i \in I\right\}$. It is easy to see by induction in the complexity of formulae that for every positive formula (built up from conjunctions and disjunctions only) $\Phi\left(x_{1}, \ldots, x_{n}\right)$ and all $\bar{a}_{1}, \ldots, \bar{a}_{n}$ with $a_{r}$ in $\mathfrak{B}_{i}$.

$$
I \models \Phi\left(\bar{a}_{1}, \ldots, \bar{a}_{n}\right) \quad \text { if and only if } \exists k \in \sum \mathfrak{B}_{k} \models \Phi\left(f_{1, k}\left(a_{1}\right), \ldots . f_{\text {ink }}\left(a_{n}\right)\right)
$$

For a tomic formulas it is just the definition. The inductive step for $\wedge$ and $\vee$ is trivial from right to left. From left to right follows from the directed property of $\sum$ and the fact that positive sentences are preserved by homomorphisms. Suppose now that $\Phi$ and $\Theta$ are positive and $\mathfrak{B}_{i} \models \forall x_{1} \ldots \forall x_{n}\left(\Phi \rightarrow \exists y_{1} \ldots \exists y_{i} \Theta\right)$ for every $i \in \sum$. Suppose $\mathfrak{D} \models \Phi\left(\bar{a}_{1}, \ldots, \bar{a}_{n}\right)$ with $a_{r}$ in $\mathfrak{B}_{i_{r}}$ then

$$
\mathfrak{B}_{k} \vDash \Phi\left(f_{i, k}\left(a_{1}\right), \ldots, f_{i, k}\left(a_{n}\right)\right)
$$


for some $k$ and so

$$
\mathfrak{B}_{k}=\Theta\left(f_{i, k}\left(a_{1}\right), \ldots, f_{i, k}\left(a_{n}\right), b_{1}, \ldots, b_{\imath}\right)
$$

for some $b_{1}, \ldots, b_{t}$ in $\mathfrak{B}_{k}$, Since $\bar{b}_{r}=f_{k k}\left(b_{r}\right)$ we have by (2):

$$
\mathcal{D}=\Theta\left(\bar{a}_{1}, \ldots, \bar{a}_{n}, \bar{b}_{1}, \ldots, \bar{b}_{t}\right) \text {. }
$$

Hence,

$$
\widetilde{C} \models \forall x_{1} \ldots \forall x_{n}\left[\Phi\left(x_{1}, \ldots, x_{n}\right) \rightarrow \exists y_{1} \ldots \exists y_{t}\left(\Theta\left(x_{1}, \ldots, x_{n} y_{1}, \ldots, y_{t}\right)\right] .\right.
$$

Since universal-Horn formulas belong to the family described in Corollary 5 , and since equations are universal-Horn formulas, we have :

COROllary 6. The subdirect decomposition theorem holds for quasivarieties. In particular, it holds for varicties (Birkhoff).

Rrmarks. Note that in Theorem 4 or Corollary 5 the class is not required to be closed under products; therefore, the decomposition may occur "outside" of the class but each factor of the product is in the class. Malcev (1971) has shown that a class $K$ is a quasivariety if and only if it is closed under direct limits, products, and substructures (see Ek lof (1975) for an elegant proof), in this case the decomposition occurs 'inside'. All the results in this paper may be extended to classes of many sorted structures closed under direct limits, the modifications are trivial.

\section{References}

G. Birkhoff (1944), "Subdirect unions in universal algebra", Bull. Amer. Math. Soc. 50, 764-768.

C. C. Chang and H. J. Keisler 1973). Model theory (North Holland, Amsterdam).

P. M. Cohn (1965), Unilersal alochra (Harper and Row. New York).

P. C. Ekkof(1975), 'Categories of local functors'. Model theory and algebra, pp. $91-116$ (Lecture Notes in Mathematics 498. Springer-licrlag. New York).

G. Grätzer 11968). Liniersal alud (Van Nostrand, New York).

P. R. Halmos (1962), Alqebraic legic (Chelsea, New York).

A. I. Makev (1971). The metamahematics of algebraic systems (North Holland, Amsterdam).

R. S. Pierce (1968). Introduction to the theory of abstract algebras (Holt, Rinehart and Winston. New York).

H. Rasiowa (1974). In alyebrai approach to non-classical logics (North Holland. Amsterdam).

Department of Mathematics

Universidad de los Ande,

Apartado Aéreo 4976

Bogotá. D. E.

Colombia 